\title{
Jean-Baptiste Biot
}

Astronome, mathématicien, physicien aux multiples centres d'intérêt, professeur au Collège de France à la chaire de physique mathématique, Jean-Baptiste Biot est le premier à établir l'origine extra-terrestre des météorites. Il est surtout connu pour ses travaux sur le magnétisme (loi de Biot-Savart) et, en optique, sur la rotation du plan de polarisation de la lumière lors de la traversée d'une solution.

Jisinat

ean-Baptiste Biot naît le 21 avril 1774 à Paris, et grandit dans 'ambiance à la fois instable et formidablement bouillonnante de la Révolution. Son père Joseph Biot, issu d'une longue lignée de paysans lorrains, est le premier à s'émanciper du monde agricole ; il est recruté au Trésor, et assure à la maisonnée un train de vie confortable. II nourrit pour son fils l'ambition d'une carrière dans le commerce.

Le jeune Jean-Baptiste est un élève doué pour les sciences, formé à Louis-Le-Grand. À la fin de son cursus, en 1791, il complète sa formation en mathématiques par des cours privés de AntoineRené Mauduit [1731-1815], alors professeur au Collège de France. Puis il se rend au Havre, où il se met au service d'un marchand, en tant qu'assistant-clerc. II s'y ennuie tant qu'il choisit de s'enrôler dans l'armée, et intègre le régiment d'artillerie de I'armée du Nord en septembre 1792. II participe à la bataille de Hondschoote, qui brise le siège de Dunkerque en septembre 1793. Mais, malade, il décide de quitter l'armée et de rentrer chez ses parents. Sur la route qui le ramène à Paris, toujours en uniforme, il est arrêté, accusé de désertion et mené devant le comité de surveillance révolutionnaire - un bien mauvais pas, dont il ne se sort que de justesse.

\section{Les années d'étude}

La convalescence de Biot dure plusieurs mois, qu'il met à profit pour se perfectionner en mathématiques. En janvier 1794, il est reçu à l'examen d'entrée de la prestigieuse École des Ponts et Chaussées. Cette même année, la Convention inaugure l'École centrale des Travaux Publics de Paris (future École polytechnique) afin de former rapidement les ingénieurs et les cadres supérieurs que les universités, alors fermées par décret, ne peuvent plus fournir. Biot se joint à la première fournée d'étudiants sous la férule, entre autres, de Monge [1746-1818], Lacroix [17651843] et Laplace [1749-1827].

Comme quelques camarades, Biot s'implique dans la vie politique ; une activité dangereuse dans la France révolutionnaire, d'autant qu'il est du côté des Royalistes. Lors d'une insurrection contre la Convention, il est arrêté et ne doit sa relaxe (probablement sa survie!) qu'à l'intervention de Monge. II peut retourner sur les bancs et achève brillamment ses études en 1797. II souhaite enseigner. Or, sous l'impulsion de Lakanal [1762-1845], la Convention a instauré en 1795 la création d'une école centrale (futur lycée) dans chaque département de France. Une position de professeur en mathématiques s'ouvre

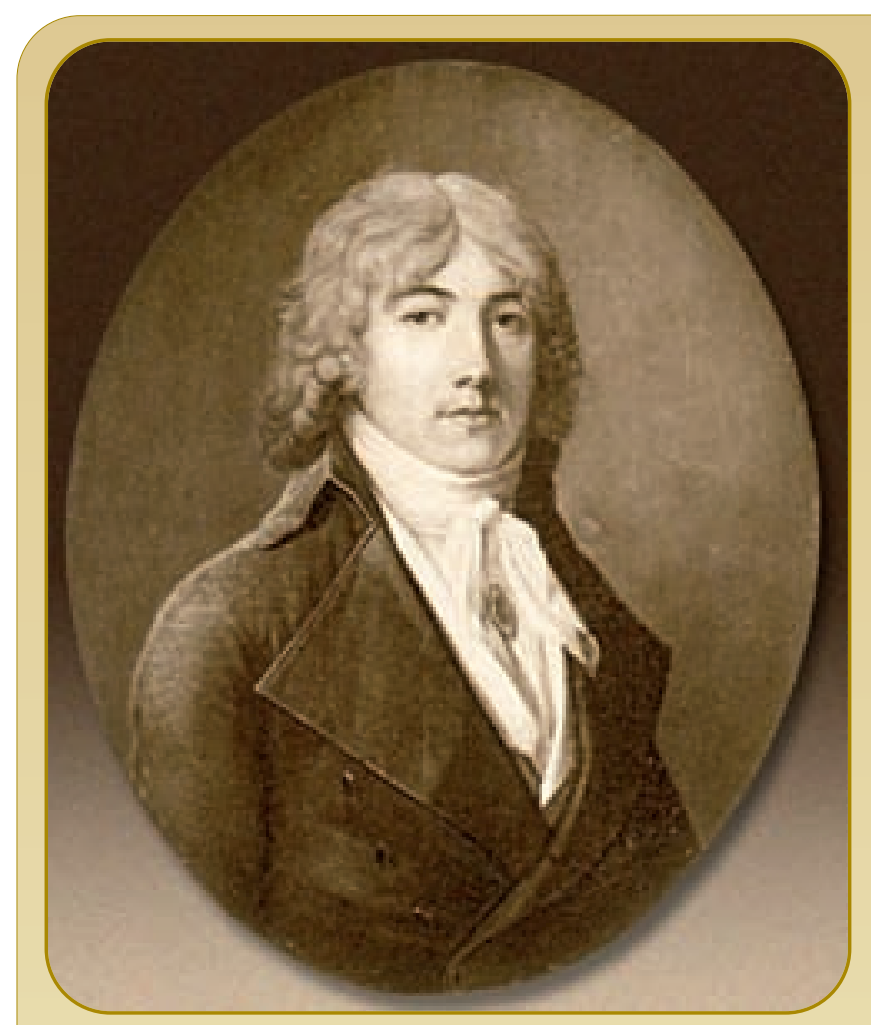

Principales dates

\section{1 avril 1774}

Naissance à Paris (France)

1801 Chaire de physique mathématique au Collège de France

1803 Enquête sur la météorite de L'Aigle

1804 Première ascension en ballon avec Gay-Lussac

1809 Chaire d'astronomie de la Faculté des Sciences de Paris

1820 Loi de Biot-Savart

1840 Rumford medal pour la Loi de polarisation rotatoire

\section{3 février 1862}

Mort à Paris (France)

à celle de Beauvais et Biot, grâce à l'appui de son professeur Lacroix, décroche le poste. Il est également nommé examinateur d'admission à l'École polytechnique en 1799. Ce Parisien garde ainsi un lien fort avec la capitale. Cette même année, il se lie avec Laplace et convainc le maître de lui confier la relecture de sa monumentale Mécanique Céleste. C'est un travail de Titan, qui dure deux ans et l'épuise, mais dont il s'acquitte honorablement. Et puis, quel formidable apprentissage ! L'aventure a également rapproché les deux hommes, et Biot, au-delà 
de l'enseignement du livre, bénéficie désormais du soutien et de la caution scientifique de Laplace.

En s'installant à Beauvais, Biot se rapproche d'un camarade de promotion, Barnabé Brisson, dont il épouse la sœur Gabrielle en 1797. Ils ont un fils, Edouard Constant, né en 1803. Gabrielle est une jeune fille de haute instruction; Biot la familiarise aux mathématiques et à la physique, et elle se charge, pour lui, de traduire de l'allemand le livre de Ernst Gottfried Fischer [17561831], qui est publié sous le titre Physique Mécanique en 1806.

\section{Travaux mathématiques}

Biot confirme très vite son potentiel en mathématiques, et acquiert une maturité fertile dans le domaine, notamment en mathématiques appliquées à la physique et en géométrie. II présente à Laplace sa solution d'un problème de géométrie analytique soulevé par le mathématicien suisse Euler [1707-1783], et irrésolu depuis. Laplace, séduit, organise un exposé à l'Académie des Sciences. Monge, le général Bonaparte et Lagrange [1736-1813] sont présents. Premier succès. Plus tard, selon la légende, Laplace montrera à Biot une liasse de papiers jaunis couverts de son écriture qui indiquent que, plus jeune, il s'était attaqué au même problème avec une méthode similaire mais sans aller au bout de l'exercice...

Les nominations s'enchaînent. Biot est élu associé non résident de l'Académie des Sciences le 25 mai 1800. II est appelé à la chaire de physique mathématique en novembre 1801 au Collège de France, à la suite de Cousin [1739-1800] qui occupait celle de physique générale. En avril 1803, suite à la réorganisation de l'Académie des Sciences, Delambre [1749-1822] devient secrétaire perpétuel et libère un fauteuil en section des mathématiques; Biot, bien qu'il se soit orienté vers la physique expérimentale et que sa production mathématique soit peu fournie depuis 1800 , parvient néanmoins à emporter les élections. II a 29 ans.

\section{Activités expérimentales}

Il entame alors une série de campagnes expérimentales. En juin 1803, à la demande du ministre de l'Intérieur Chaptal [17561832], il se rend à L'Aigle, dans l'Orne, où une météorite est tombée le 26 avril, et fait un rapport considéré comme la première démonstration de l'origine non terrestre des météorites. Le 24 août 1804, il accompagne Gay-Lussac [1778-1850] dans le premier vol en ballon à but scientifique ; ils entament leur ascension à partir des jardins du Conservatoire des Arts, et montent jusqu'à près de 4000 mètres pour l'étude de la variation de l'intensité du magnétisme terrestre et des propriétés chimiques et électriques de l'atmosphère avec l'altitude.

Puis Biot entame une collaboration fertile avec Arago [17861853], un jeune prodige tout juste admis à l'École polytechnique. Ensemble, ils mettent au point un appareil (le Fortuna) qui permet de mesurer la densité des gaz et qui servira aux travaux de J.-B. Dumas [1800-1884]. Surtout, ils sont mandatés en $\gg \gg$

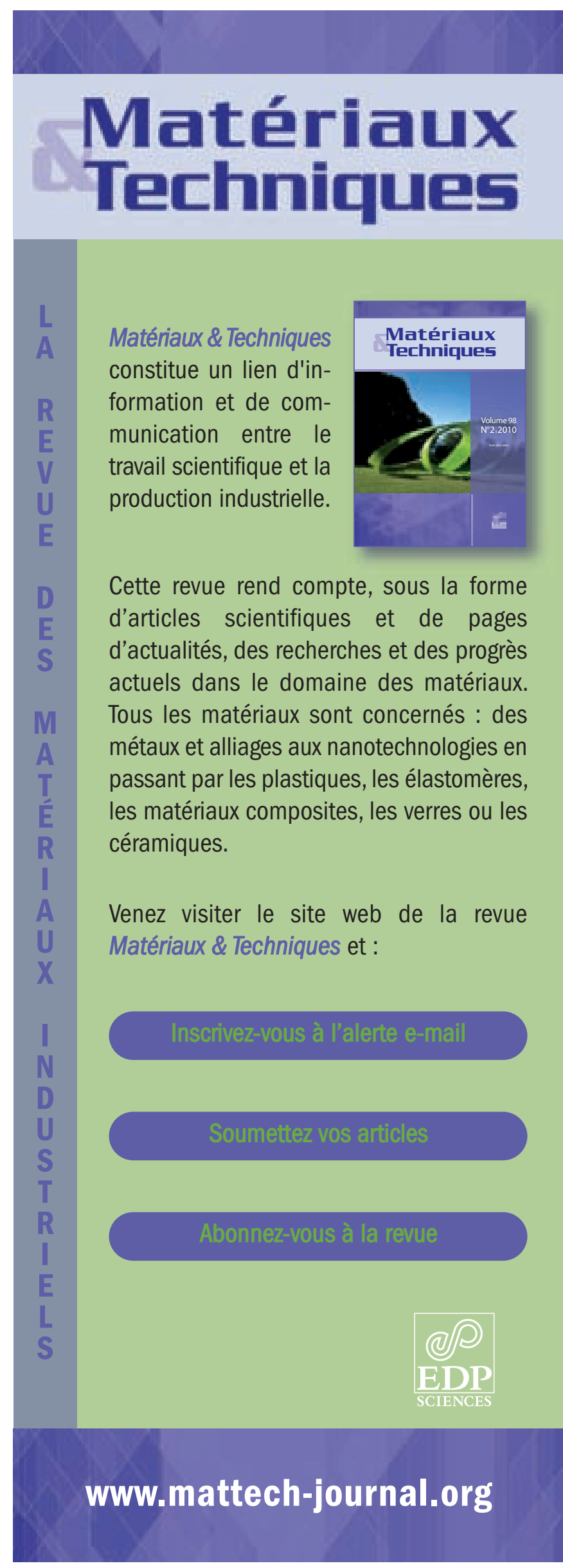


1804 par le Bureau des Longitudes pour la mesure du méridien de Paris. En effet Méchain [1744-1804], qui dirigeait l'expédition précédente, est mort sans avoir pu achever sa mission. Biot et Arago s'installent à l'Observatoire de Paris, et y passent dixhuit mois à acquérir les techniques nécessaires aux expériences prévues. Car leur voyage doit servir, outre à la mesure de la méridienne, un second objectif, tout aussi ambitieux : la mesure de la gravité à l'aide d'un pendule, afin de préciser la forme du globe terrestre.

En 1806, Biot est nommé astronome assistant au Bureau des Longitudes. Le 3 septembre, ils partent pour l'Espagne, rejoignent Barcelone, triangulent la côte Catalane, passent par Ibiza, arrivent enfin dans l'Île de Majorque. Cette étape est stratégique, car Majorque permet de prolonger le méridien de Paris bien plus au sud que Barcelone. Mais en 1808, Napoléon annexe une partie du territoire espagnol, et déclenche la guerre d'Indépendance. II ne fait pas bon être Français dans les Baléares à ce moment-là. Avec leur attirail scientifique et leurs instruments de mesure sophistiqués, Biot et Arago ont tout l'air de parfaits espions. Chaque jour de présence supplémentaire est un jour de trop. Biot, prudent, décide de retourner en France, tandis que Arago s'entête : il ira jusqu'au bout, au terme d'une aventure qui lui conférera une aura extraordinaire.

De retour à Paris, Biot s'embarque avec Claude-Louis Mathieu [1783-1875] pour une série de mesures précises de la gravité terrestre le long du méridien, de Bordeaux à Dunkerque. C'est un travail minutieux et considérable, récompensé par l'Académie des Sciences, et qui vaut probablement à Biot d'être appelé à la chaire d'astronomie de la Faculté des Sciences de Paris à sa création en 1809 - une position qu'il occupera près de 40 ans. Il obtient un doctorat ès sciences en août de la même année.

\section{Magnétisme et Optique}

Éclectique pour tout ce qui concerne le savoir scientifique, Biot est aussi passionné d'histoire et de géographie. Il étudie le calendrier des Égyptiens, fait des recherches sur l'astronomie chinoise, s'intéresse à la vie des grands hommes de science. Il publie ainsi plusieurs ouvrages sur le sujet, parmi lesquels Essai sur l'histoire générale des sciences pendant la Révolution française (1803) ou Études sur l'astronomie indienne et l'astronomie chinoise (1862)... II rédige également la première biographie officielle de Newton, dressant un portrait sans concession du grand homme.

L'étude de la lumière est probablement le domaine où Biot s'est le plus impliqué. Pourtant c'est en magnétisme qu'il laisse d'abord son empreinte : avec Savart [1791-1841], ils découvrent en 1820 que le champ magnétique créé par un courant circulant dans un fil est inversement proportionnel à la distance à ce fil, et établissent la loi (fondamentale en magnétostatique) qui porte leur nom.

Dans les années 1820 Biot est, avec Arago, la référence de I'optique en France. Leur rivalité, naturellement forte, s'accentue encore lors des élections de 1830 au poste de secrétaire per- pétuel de l'Académie des Sciences - qu'Arago remporte. Elle se cristallise ensuite sur les techniques de photographie, Arago soutenant sans réserve le daguerréotype et Biot se faisant le champion du procédé de Talbot, dont il juge le principe plus riche.

Biot s'intéresse longuement aux phénomènes de polarisation de la lumière. II a l'idée de les corréler à l'état de cristallisation des corps, et il établit en 1835 avec Jean-François Persoz [18051868] les lois de la polarisation rotatoire lors de la traversée d'un milieu. Pour ses expériences, il invente le polarimètre, parfois appelé saccharimètre car on l'utilise pour mesurer la concentration en sucre d'une solution. Ses travaux inspireront notamment Pasteur [1822-1895], qui démontrera le lien entre la symétrie des molécules et leur pouvoir rotatoire. Biot reçoit en 1840 la prestigieuse Rumford Medal de la Royal Society of London "for his researches in, and connected with, the circular polarization of light».

\section{Les honneurs}

Jean-Baptiste Biot est membre associé de plusieurs Académies étrangères, dont la Royal Society of London dès 1815. Membre titulaire du Bureau des Longitudes en 1825, il en devient président en 1835. Il est élu à l'Académie des Inscriptions et BellesLettres en 1841 et à l'Académie française en 1856.

Par ailleurs, il est fait Chevalier (en 1814) puis Commandeur (en 1849) de la Légion d'honneur. II est doyen de la Faculté des Sciences de Paris de 1840 à 1841, succédant à Thénard [17771857] et Poisson [1781-1840].

Ses contemporains dépeignent un homme curieux, fin, inventif (selon Sainte-Beuve), mais également vaniteux et partial dans son jugement (selon Olinthus Gregory). II n'empêche : Biot survit à tous les régimes politiques. II rend à Pasteur et à d'autres le service que lui ont rendu Laplace et Lacroix - soutenant efficacement les jeunes esprits qui l'impressionnent et les introduisant dans les meilleurs cercles scientifiques. II incarne ainsi fidèlement, et jusqu'au bout, l'idéal de Monge et Sadi Carnot [1796-1832] qui, à la fin du $18^{\mathrm{e}}$ siècle, créèrent les conditions d'émergence d'une promotion exceptionnelle de jeunes gens au service à la fois de la République et d'une idée bien particulière d'un mieux-être par le progrès scientifique. II rend l'âme le 3 février 1862, à l'âge de 88 ans, au Collège de France.

\section{Riad HAIDAR \\ Onera}

haidar@onera.fr

\section{Références}

[1] Biographie de J.-B. Biot sur www.pasteurbrewing.com

[2] E. Picard, La vie et l'œuvre de Jean-Baptiste Biot, lu à I'Institut de France le 12 décembre 1927. 